\title{
Statistical Inference for a Two Station Tandem Queue with Atmost One Customer to Wait Betweenthe Stations
}

\author{
P. Chandrasekhar \\ Department of Statistics \\ Loyola College, Chennai, India \\ drchandrasekharin@yahoo.co.in \\ V.S. Vaidyanathan \\ Department of Statistics \\ Pondicherry University, India \\ vaidya.stats@gmail.com
}

\begin{abstract}
A maximum likelihood estimator (MLE), a consistent asymptotically normal (CAN) estimator and asymptotic confidence limits for the expected number of customers in the system for a sequential two station, single server system with Poisson input and exponential service, where no queue is allowed in front of station 1 and atmost one customer is allowed to wait between the stations and with blocking are obtained.
\end{abstract}

Keywords: CAN estimator- expected number of customers in the system maximum likelihood estimator - multivariate central limit theorem-Slutsky theorem-tandem queue.

\section{Introduction}

Most of the studies on several queueing models are confined to only obtaining expressions for transient or stationary (steady state) solutions and do not consider the associated statistical inference problems. Parametric estimation, Interval estimation and Bayesian estimation are some of the statistical tools to understand any random phenomena using stochastic models. Analysis of queueing systems in this direction has not received due attention in the past. Whenever the systems are fully observable in terms of their random components such as inter-arrival and service times, standard parametric techniques of statistical theory are quite appropriate. Bhat (2003) has provided an overview of methods available for estimation, when the information is restricted to the number of customers in the system at some discrete points in time. Bhat (2003) has also described how maximum likelihood estimation is applied directly to the underlying Markov chain in the queue length process in $\mathrm{M} / \mathrm{G} / 1$ and $\mathrm{GI} / \mathrm{M} / 1$ queues. Table 1 indicates the present state of work of queueing systems, wherein the asymptotic confidence limits for measures of system performance such as (i) $L_{S}$, the expected number of customers in the system, (ii) $W_{Q}$, the mean waiting time in the queue and (iii) $W_{S}$, the mean waiting time in the system and so on are obtained. 
Table1: Present state of work of Queueing systems

\begin{tabular}{|c|c|c|c|}
\hline $\begin{array}{c}\text { Serial } \\
\text { number }\end{array}$ & System Description & Authors & $\begin{array}{c}\text { Confidence } \\
\text { limits } \\
\text { obtained for }\end{array}$ \\
\hline 1 & $\mathrm{M} / \mathrm{M} / 1 / 0$ and $\mathrm{M} / \mathrm{M} / 1 / \mathrm{N}$ & Yadavalli et. al (2004) & $\mathrm{W}_{\mathrm{Q}}$ \\
\hline 2 & $\mathrm{M} / \mathrm{M} / \mathrm{c} / 0$ and M/M/c/N & Yadavalli et. al (2006) & $\mathrm{W}_{\mathrm{Q}}$ \\
\hline 3 & $\begin{array}{c}\text { Tandem queue with } \\
\text { blocking and } \\
\text { dependent structure for } \\
\text { service times }\end{array}$ & $\begin{array}{c}\text { Chandrasekhar et.al } \\
(2006)\end{array}$ & $\mathrm{L}_{\mathrm{S}}, \mathrm{W}_{\mathrm{S}}$ \\
\hline 4 & $\begin{array}{c}\text { Tandem queue with } \\
\text { blocking }\end{array}$ & $\begin{array}{c}\text { Chandrasekhar et.al } \\
(2008)\end{array}$ & $\mathrm{W}_{\mathrm{S}}$ \\
\hline 5 & $\begin{array}{c}\text { Two station and three } \\
\text { station tandem queues } \\
\text { with blocking }\end{array}$ & $\begin{array}{c}\text { Chandrasekhar et.al } \\
(2009)\end{array}$ & $\mathrm{L}_{\mathrm{S}}, p_{a}$ \\
\hline
\end{tabular}

Generally speaking, the queueing models assume that each service channel consists of only one station. Situations do exist, where each service channel may consist of several stations in series. Here, a customer must pass through successively all the stations before completing his service. Such situations are known as queues in series or tandem queues. The following are some examples of tandem queues:

a) In a manufacturing process, units must pass through a series of service channels (work stations), where each service channel performs a given task or job.

b) In a university registration process, each registrant must pass through a series of counters such as advisor, department chairman (Head of the Department), cashier etc.

c) In a clinical physical examination procedure, a patient goes through a series of stages such as lab tests, ECG, chest X-ray etc.

In all these model structures, it is not only sufficient to know how many persons are there in the system but also where they are.

An attempt is made in this paper to study in detail a sequential two station single server system with Poisson input and exponential service, where no queue is allowed in front of station 1 and atmost one customer is allowed to wait between the stations. A MLE, CAN estimator and asymptotic confidence limits for the expected number of customers in the system are obtained. The system description and assumptions are given in the next section. 


\section{System description and assumptions}

Consider a simplified one channel queueing system consisting of two servicestationsas below:

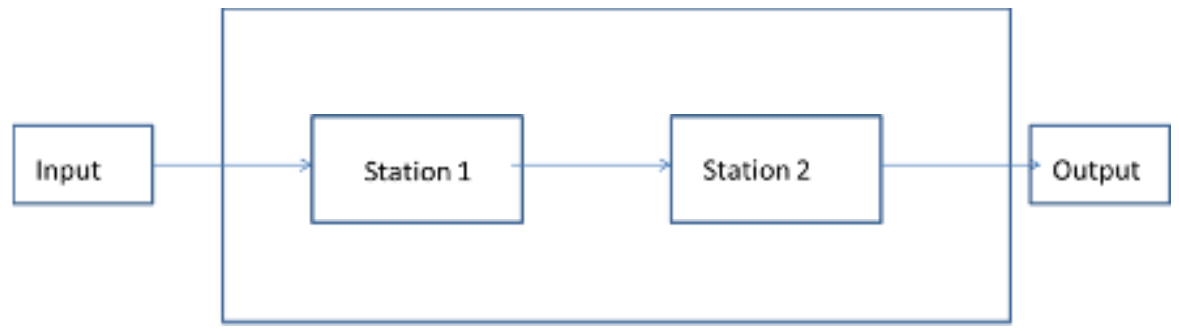

Fig 1: System configuration.

A customer arriving for service must go through station 1 and station 2 before completing his service. The assumptions of the model are as follows:

I. Arrivals occur according to Poisson distribution with mean rate $\lambda$ for service at station1.

II. Service times at each station are exponentially distributed with the same service rate $\mu$

III. No queue is allowed in front of station 1 and atmost one customer is allowed to wait between station 1 and station 2 .

IV. Each station is either free or busy.

V. Station 1 is said to be blocked, if the customer in station 1 completes his service beforestation 2 becomes free and there is a customer waiting between the stations.

\section{Analysis of the system}

Let the symbols 0,1 and $b$ represent free, busy and blocked states of a station. Let $X(t), Y(t)$ and $Z(t)$ respectively denote the state of station 1, the number of customers waiting between station 1 and station 2 and the state of station 2 at time $t$. The vector process $V(t)=\{(X(t), Y(t), Z(t)), t \geq 0\}$ with state space

$$
E=\{(0,0,0),(0,0,1),(0,1,1),(1,0,0),(1,0,1),(1,1,1),(b, 1,1)\}
$$

denotes the state of the system at time $t$. Since the interarrival and service times are exponential, it follows that the process $V(t)$ is a Markov Process with the infinitesimal generator $Q$ given by $Q=$

$$
(0,0,0) \quad(1,0,0) \quad(0,0,1) \quad(1,0,1) \quad(0,1,1) \quad(b, 1,1) \quad(1,1,1)
$$

$(0,0,0)$
$(1,0,0)$
$(0,0,1)$
$(1,0,1)$
$(0,1,1)$
$(b, 1,1)$
$(1,1,1)$$\left[\begin{array}{ccccccc}-\lambda & \lambda & 0 & 0 & 0 & 0 & 0 \\ 0 & -\mu & \mu & 0 & 0 & 0 & 0 \\ 0 & 0 & -(\lambda+\mu) & \lambda & 0 & 0 & 0 \\ 0 & 0 & 0 & -2 \mu & \mu & 0 & 0 \\ 0 & 0 & 0 & 0 & -\lambda & 0 & \lambda \\ 0 & 0 & \mu & 0 & \mu & -2 \mu\end{array}\right]$




\section{P. Chandrasekhar, V.S. Vaidyanathan}

Let $p_{i j k}(t)=\operatorname{Pr}[V(t)=(i, j, k)] \forall(i, j, k) \in E$ represent the probability that the system is in state $(i, j, k)$ at time ' $\mathrm{t}$ ' with the initial condition $p_{000}(0)=1$. From the infinitesimal generator given in (3.2), we have the following system of differential - difference equations:

$$
\begin{aligned}
& \frac{d p_{000}(t)}{d t}=-\lambda p_{000}(t)+\mu p_{001}(t) \\
& \frac{d p_{100}(t)}{d t}=\lambda p_{000}(t)-\mu p_{100}(t)+\mu p_{101}(t) \\
& \frac{d p_{001}(t)}{d t}=\mu p_{100}(t)-(\lambda+\mu) p_{001}(t) \\
& \frac{d p_{101}(t)}{d t}=\lambda p_{001}(t)-2 \mu p_{101}(t)+\mu p_{111}(t) \\
& \frac{d p_{011}(t)}{d t}=\mu p_{101}(t)-\lambda p_{011}(t)+\mu p_{b 11}(t) \\
& \frac{d p_{b 11}(t)}{d t}=-\mu p_{b 11}(t)+\mu p_{111}(t) \\
& \frac{d p_{111}(t)}{d t}=\lambda p_{011}(t)-2 \mu p_{111}(t)
\end{aligned}
$$

\subsection{Transient Solution}

The system of equations (3.3) - (3.9) along with the equation $\sum_{(i, j, k) \in E} p_{i j k}(t)=1$ can be solved using Laplace transforms. Let $L_{i}(s)$ be the Laplace transform of $p_{i}(t)$. By taking $(0,0,0)=a,(1,0,0)=b,(0,0,1)=c,(1,0,1)=d,(0,1,1)=e,(b, 1,1)=f \quad$ and $(1,1,1)=g$ and taking Laplace transform on both the sides of (3.3) - (3.9) and solving for $L_{i}(s)$ using $\sum_{(i, j, k) \in E} p_{i j k}(t)=1$, we get

$$
\begin{aligned}
& L_{a}(s)=\frac{\prod_{j=1}^{3}\left(s-\alpha_{j}\right)}{\prod_{j=1}^{4}\left(s-\gamma_{j}\right)}+\frac{\lambda \mu^{5}(s+\mu)}{\prod_{j=1}^{4}\left(s-\gamma_{j}\right) \prod_{j=1}^{3}\left(s-\beta_{j}\right)} L_{d}(s) \\
& L_{b}(s)=\frac{(s+\lambda+\mu)}{\lambda \mu} \frac{\prod_{j=1}^{4}\left(s-\delta_{j}\right)}{\prod_{j=1}^{3}\left(s-\beta_{j}\right)} L_{d}(s) \\
& L_{c}(s)=\frac{\prod_{j=1}^{4}\left(s-\delta_{j}\right)}{\lambda \prod_{j=1}^{3}\left(s-\beta_{j}\right)} L_{d}(s)
\end{aligned}
$$




$$
\begin{aligned}
& L_{e}(s)=\frac{\mu(s+\mu)(s+2 \mu)}{\prod_{j=1}^{3}\left(s-\beta_{j}\right)} L_{d}(s) \\
& L_{f}(s)=\frac{\lambda \mu^{2}}{\prod_{j=1}^{3}\left(s-\beta_{j}\right)} L_{d}(s) \\
& L_{g}(s)=\frac{\lambda \mu(s+\mu)}{\prod_{j=1}^{3}\left(s-\beta_{j}\right)} L_{d}(s)
\end{aligned}
$$

By taking inverse Laplace transform on both sides of (3.10) - (3.15), we get expressions for $p_{i j k}(t)$. It may be noted that

$\alpha_{1}, \alpha_{2}$ and $\alpha_{3}$ are the roots of $s^{3}+(\lambda+4 \mu) s^{2}+\left(3 \lambda \mu+5 \mu^{2}\right) s+\mu^{2}(\lambda+2 \mu)=0$;

$\beta_{1}, \beta_{2}$ and $\beta_{3}$ are the roots of $s^{3}+(\lambda+3 \mu) s^{2}+\left(3 \lambda \mu+2 \mu^{2}\right) s+\lambda \mu^{2}=0$;

$\gamma_{1}, \gamma_{2}, \gamma_{3}$ and $\gamma_{4}$ are the roots of

$s^{4}+(2 \lambda+4 \mu) s^{3}+\left(\lambda^{2}+7 \lambda \mu+5 \mu^{2}\right) s^{2}+\left(5 \lambda \mu^{2}+3 \lambda^{2} \mu+2 \mu^{3}\right) s+\lambda^{2} \mu^{2}=0$;

$\delta_{1}, \delta_{2}, \delta_{3}$ and $\delta_{4}$ are the roots of

$s^{4}+(\lambda+5 \mu) s^{3}+\left(8 \mu^{2}+5 \lambda \mu\right) s^{2}+\left(6 \lambda \mu^{2}+4 \mu^{3}\right) s+\lambda \mu^{3}=0$.

\subsection{Steady State Solution}

Since we wish to study the stationary behaviour of the system, let $\lim _{t \rightarrow \infty} p_{i j k}(t)=p_{i j k}$. Let $\underline{p}=\left(p_{000}, p_{001}, p_{011}, p_{100}, p_{101}, p_{111}, p_{b 11}\right) \quad$ be the stationary distribution corresponding to the Markov Process $V(t)$. Allowing $t \rightarrow \infty$ on both the sides of (3.3) - (3.9), noting that $\lim _{t \rightarrow \infty} \frac{d p_{i j k}(t)}{d t}=0$ and solving for $p_{i j k}$, we obtain the steady state probabilities as follows:

$$
\begin{aligned}
& p_{000}=\frac{\mu^{2}}{\left(3 \lambda^{2}+4 \lambda \mu+2 \mu^{2}\right)} \\
& p_{100}=\frac{\mu(\lambda+\mu)}{\left(3 \lambda^{2}+4 \lambda \mu+2 \mu^{2}\right)} \\
& p_{001}=\frac{\lambda \mu}{\left(3 \lambda^{2}+4 \lambda \mu+2 \mu^{2}\right)} \\
& p_{101}=\frac{\lambda^{2}}{\left(3 \lambda^{2}+4 \lambda \mu+2 \mu^{2}\right)}=p_{b 11}=p_{111} \\
& p_{011}=\frac{2 \lambda \mu}{\left(3 \lambda^{2}+4 \lambda \mu+2 \mu^{2}\right)}
\end{aligned}
$$




\subsection{Expected number of customers in the system}

The expected number of customers in the system is given by

$$
\begin{aligned}
& L_{s}=\sum_{n=0}^{\infty} n p_{n}=\left(p_{001}+p_{100}\right)+2\left(p_{101}+p_{011}\right)+3\left(p_{b 11}+p_{111}\right) \\
& =\frac{\left(8 \lambda^{2}+6 \lambda \mu+\mu^{2}\right)}{\left(3 \lambda^{2}+4 \lambda \mu+2 \mu^{2}\right)}
\end{aligned}
$$

In the next section, we obtain maximum likelihood and consistent asymptotically normal estimators for the expected number of customers in the system.

\section{MLE and CAN estimator for the expected number of customers in the system}

\subsection{ML estimator}

Let $X_{1}, X_{2}, \ldots, X_{n}$ and $Y_{1}, Y_{2}, \ldots, Y_{n}$ be random samples of size n, each drawn from exponential interarrival time and exponential service time populations with the parameters $\lambda$ and $\mu$ respectively. It is clear that $E(\bar{X})=\frac{1}{\lambda}$ and $E(\bar{Y})=\frac{1}{\mu}$, where $\bar{X}$ and $\bar{Y}$ are the sample means of interarrival times and service times respectively. It can be shown that $\bar{X}$ and $\bar{Y}$ are the MLEs of $\frac{1}{\lambda}$ and $\frac{1}{\mu}$ respectively.

Let $\theta_{1}=\frac{1}{\lambda}$ and $\theta_{2}=\frac{1}{\mu}$. Clearly, the expected number of customers in the system given in (3.21) reduces to

$$
L_{s}=\frac{\left(\theta_{1}^{2}+6 \theta_{1} \theta_{2}+8 \theta_{2}^{2}\right)}{\left(2 \theta_{1}^{2}+4 \theta_{1} \theta_{2}+3 \theta_{2}^{2}\right)}
$$

and hence the MLE of $L_{s}$ is given by

$$
\hat{L}_{s}=\frac{\left(\bar{X}^{2}+6 \bar{X} \bar{Y}+8 \bar{Y}^{2}\right)}{\left(2 \bar{X}^{2}+4 \bar{X} \bar{Y}+3 \bar{Y}^{2}\right)}
$$

It may be noted that $\hat{L}_{s}$ given in (4.2) is a real valued function in $\bar{X}$ and $\bar{Y}$, which is also differentiable. Consider the following application of multivariate central limit theorem (Radhakrishna Rao, 1974).

\subsection{Application of Multivariate Central Limit Theorem}

Suppose $\mathrm{T}_{1}^{\prime}, \mathrm{T}_{2}^{\prime}, \mathrm{T}_{3}^{\prime}, \ldots$ are independent and identically distributed $\mathrm{k}$-dimensional random variables such that $\mathrm{T}_{\mathrm{n}}^{\prime}=\left(\mathrm{T}_{1 \mathrm{n}}, \mathrm{T}_{2 \mathrm{n}}, \mathrm{T}_{3 \mathrm{n}}, \ldots, \mathrm{T}_{\mathrm{kn}}\right), \mathrm{n}=1,2,3, \ldots$ having the first 
and second order moments $E\left(T_{n}\right)=\mu$ and $\operatorname{var}\left(T_{n}\right)=\sum$. Define the sequence of random variables $\overline{\mathrm{T}}_{\mathrm{n}}{ }^{\prime}=\left(\overline{\mathrm{T}}_{1 \mathrm{n}}, \overline{\mathrm{T}}_{2 \mathrm{n}}, \overline{\mathrm{T}}_{3 \mathrm{n}}, \ldots, \overline{\mathrm{T}}_{\mathrm{kn}}\right), \mathrm{n}=1,2,3, \ldots$ where

$\overline{\mathrm{T}}_{\mathrm{in}}=\frac{\sum_{\mathrm{j}=1}^{\mathrm{n}} \mathrm{T}_{\mathrm{ij}}}{\mathrm{n}}, \mathrm{i}=1,2,3, \ldots, \mathrm{k}$.

Then, $\sqrt{\mathrm{n}}\left(\overline{\mathrm{T}}_{\mathrm{n}}-\mu\right) \stackrel{\mathrm{d}}{\longrightarrow} \mathrm{N}_{\mathrm{k}}\left(0, \sum\right)$, as $\mathrm{n} \rightarrow \infty$.

\subsection{CAN Estimator}

By applying the multivariate central limit theorem given in section 4.2 , it readily follows that

$\sqrt{\mathrm{n}}\left[(\overline{\mathrm{X}}, \overline{\mathrm{Y}})-\left(\theta_{1}, \theta_{2}\right)\right] \stackrel{\mathrm{d}}{\longrightarrow} \mathrm{N}_{2}(0, \Sigma)$ as $\mathrm{n} \rightarrow \infty$.

The dispersion matrix $\sum=\left(\left(\sigma_{\mathrm{ij}}\right)\right)$ is given by $\sum=\operatorname{diag}\left(\theta_{1}^{2}, \theta_{2}^{2}\right)$.

Again from Radhakrishna Rao (1974),

We have $\sqrt{\mathrm{n}}\left(\hat{\mathrm{L}_{\mathrm{s}}}-\mathrm{L}_{\mathrm{s}}\right) \stackrel{\mathrm{d}}{\longrightarrow} \mathrm{N}\left(0, \sigma^{2}(\theta)\right)$ as $\mathrm{n} \rightarrow \infty$, where $\theta=\left(\theta_{1}, \theta_{2}\right)$ and $\sigma^{2}(\theta)=\sum_{\mathrm{i}=1}^{2}\left(\frac{\partial \mathrm{L}_{\mathrm{s}}}{\partial \theta_{\mathrm{i}}}\right)^{2} \sigma_{\mathrm{ii}}=\frac{8 \theta_{1}^{2} \theta_{2}^{2}\left(4 \theta_{1}^{2}+13 \theta_{1} \theta_{2}+7 \theta_{2}^{2}\right)^{2}}{\left(2 \theta_{1}^{2}+4 \theta_{1} \theta_{2}+3 \theta_{2}^{2}\right)^{4}}$

Hence, by applying the definition of CAN estimator, it can be seen that $\hat{\mathrm{L}}_{\mathrm{s}}$ is a CAN estimator of $L_{s}$. There are several methods for generating CAN estimators of which the Method of moments and the Method of Maximum likelihood are commonly used to generate such estimators (Sinha, 1986).

\section{Confidence interval for the expected number ofcustomers in the system}

Let $\sigma^{2}(\hat{\theta})$ be the estimator of $\sigma^{2}(\theta)$ obtained by replacing $\theta$ by a consistent estimator $\hat{\theta}$ namely $\hat{\theta}=(\bar{X}, \bar{Y})$. Let $\hat{\sigma}^{2}=\sigma^{2}(\hat{\theta})$. Since $\sigma^{2}(\theta)$ is a continuous function of $\theta, \hat{\sigma}^{2}$ is a consistent estimator of $\sigma^{2}(\theta)$. In other words, $\hat{\sigma}^{2} \stackrel{P}{\longrightarrow} \sigma^{2}(\theta) \quad$ as $\mathrm{n} \rightarrow \infty$. 
By Slutsky theorem $\left(X_{n} \stackrel{d}{\longrightarrow} X, Y_{n} \stackrel{P}{\longrightarrow} b \Rightarrow \frac{X_{n}}{Y_{n}} \stackrel{d}{\longrightarrow} \frac{X}{b}, b \neq 0\right)$, we have $\sqrt{\mathrm{n}}\left(\frac{\hat{\mathrm{L}}_{\mathrm{s}}-\mathrm{L}_{\mathrm{s}}}{\hat{\sigma}}\right) \underset{\mathrm{d}}{\longrightarrow} \mathrm{N}(0,1)$ i.e., $\operatorname{Pr}\left[-\mathrm{k}_{\frac{\sigma}{2}}<\frac{\sqrt{\mathrm{n}\left(\hat{\mathrm{L}}_{\mathrm{s}}-\mathrm{L}_{\mathrm{s}}\right)}}{\hat{\sigma}}<\mathrm{k}_{\frac{\alpha}{2}}\right]=(1-\alpha)$,

Where $\mathrm{k}_{\frac{\alpha}{2}}$ is obtained from normal tables. Hence, a $100(1-\alpha) \%$ asymptotic confidence interval for $\mathrm{L}_{\mathrm{s}}$ is given by $\hat{\mathrm{L}}_{\mathrm{s}} \pm \mathrm{k}_{\frac{\alpha}{2}} \frac{\hat{\sigma}}{\sqrt{\mathrm{n}}}$.

\section{Numerical illustration}

Table 2 and Table 3 give the 95\% confidence limits for the expected number of customers in the system by generating samples of sizes $n=200$ and $n=400$ for various values of $\lambda=0.5,1.0, \ldots, 3.0$ and $\mu=2.0,2.5, \ldots, 4.0$ from exponential distributions with means $\frac{1}{\lambda}$ and $\frac{1}{\mu}$ respectively.

Table 2: Confidence limits for expected number of customers in the system $(n=200)$

\begin{tabular}{|c|c|c|c|c|c|}
\cline { 2 - 6 } \multicolumn{1}{c|}{} & \multicolumn{5}{c|}{$\mu$} \\
\hline$\lambda$ & $\mathbf{2 . 0}$ & $\mathbf{2 . 5}$ & $\mathbf{3 . 0}$ & $\mathbf{3 . 5}$ & $\mathbf{4 . 0}$ \\
\hline $\mathbf{0 . 5}$ & $(0.8991,1.0569)$ & $(0.7990,0.9253)$ & $(0.7821,0.9026)$ & $(0.7491,0.8576)$ & $(0.6851,0.7686)$ \\
\hline $\mathbf{1 . 0}$ & $(1.1303,1.3391)$ & $(1.1081,1.3133)$ & $(0.9694,1.1458)$ & $(0.9190,1.0824)$ & $(0.8877,1.0422)$ \\
\hline $\mathbf{1 . 5}$ & $(1.4781,1.7120)$ & $(1.3498,1.5811)$ & $(1.2393,1.4622)$ & $(1.1775,1.3932)$ & $(1.1051,1.3098)$ \\
\hline $\mathbf{2 . 0}$ & $(1.6039,1.8333)$ & $(1.3922,1.6252)$ & $(1.2812,1.5081)$ & $(1.2092,1.4289)$ & $(1.1648,1.3788)$ \\
\hline $\mathbf{2 . 5}$ & $(1.6465,1.8734)$ & $(1.4916,1.7254)$ & $(1.4003,1.6335)$ & $(1.3669,1.5990)$ & $(1.1796,1.3956)$ \\
\hline $\mathbf{3 . 0}$ & $(1.7800,1.9937)$ & $(1.6139,1.8430)$ & $(1.5324,1.7653)$ & $(1.3764,1.6089)$ & $(1.3246,1.5545)$ \\
\hline
\end{tabular}


Table 3: Confidence limits for expected number of customers in the system $(n=400)$

\begin{tabular}{|c|c|c|c|c|c|}
\cline { 2 - 7 } \multicolumn{1}{|c|}{} & \multicolumn{3}{|c|}{$\mu$} \\
\hline$\lambda$ & $\mathbf{2 . 0}$ & $\mathbf{2 . 5}$ & $\mathbf{3 . 0}$ & $\mathbf{3 . 5}$ & $\mathbf{4 . 0}$ \\
\hline $\mathbf{0 . 5}$ & $(0.8930,0.9988)$ & $(0.8123,0.9004)$ & $(0.7567,0.8313)$ & $(0.7368,0.8063)$ & $(0.70461,0.7657)$ \\
\hline $\mathbf{1 . 0}$ & $(1.2225,1.3762)$ & $(1.1542,1.3012)$ & $(1.0624,1.1977)$ & $(0.9724,1.0932)$ & $(0.9094,1.0185)$ \\
\hline $\mathbf{1 . 5}$ & $(1.3361,1.4976)$ & $(1.2729,1.4307)$ & $(1.1510,1.2976)$ & $(1.1152,1.2575)$ & $(1.0316,1.1622)$ \\
\hline $\mathbf{2 . 0}$ & $(1.5490,1.7140)$ & $(1.4572,1.6225)$ & $(1.2998,1.4594)$ & $(1.2063,1.3586)$ & $(1.1440,1.2897)$ \\
\hline $\mathbf{2 . 5}$ & $(1.6493,1.8113)$ & $(1.5499,1.7149)$ & $(1.4088,1.5731)$ & $(1.3426,1.5045)$ & $(1.2221,1.3758)$ \\
\hline $\mathbf{3 . 0}$ & $(1.8519,1.9992)$ & $(1.6324,1.7951)$ & $(1.5989,1.7627)$ & $(1.5149,1.6803)$ & $(1.4126,1.5770)$ \\
\hline
\end{tabular}

It can be seen from the above tables that as the interarrival rate $\lambda$ increases (for fixed value of service rate $\mu$ ), the confidence interval for the expected number of customers in the system also increases. This means that for a fixed service rate, more the interarrival rate, more the expected number of customers in the system.Similarly, as the service rate $\mu$ increases (for fixed value of interarrival rate $\lambda$ ), the confidence interval for the expected number of customers in the system decreases. This means that for a fixed interarrival rate, more the service rate, less the expected number of customers in the system.

\section{Conclusion}

Asymptotic confidence interval for the expected number of customers in the system is studied. The expressions for transient probabilities and steady state probabilities are obtained. The results are shown numerically by generating samples of sizes 200 and 400 from exponential distributions for various values of $\lambda$ and $\mu$. It justifies that, for a fixed service rate, as the interarrival rate increases, the expected number of customers in the system increases. Also, for a fixed interarrival rate, as the service rate increases, the expected number of customers in the system decreases.

\section{Acknowledgment}

The research of the first author was sponsored by University Grants Commission, India under Emeritus Fellowship. 


\section{References}

1. Chandrasekhar, P., Chandrasekarand, B., and Yadavalli, V.S.S. (2006). Statistical Inference for a tandem queue with dependent structure for service times, Proceedings of the Sixth IASTED International Conference on Modelling, Simulation and Optimization, September 11-13,Gaborone, Botswana, 233-238.

2. Chandrasekhar, P., Natarajan, R., and Yadavalli, V.S.S. (2008). Statistical analysis for a tandem queue with blocking, Proceedings of the Second National Conference on Management Science and Practice, March 9-11,2007, Allied Publishers Pvt. Ltd, Chennai, India, 2008, 65-72.

3. Chandrasekhar,P., and Vaidyanathan,V.S. (2009). Statistical Analysis for a two station and a three station tandem queues with blocking, Journal of Applied Statistical Science, 16, 4, 425-435.

4. Narayan, U. Bhat (2003). Parameter Estimation in $M / G / 1$ and $G / / M / 1$ Queues using Queue Length Data, Stochastic Point Processes, Narosa Publishing House, New Delhi.

5. Radhakrishna Rao, C. (1974). Linear Statistical Inference and its applications, Wiley Eastern Pvt. Ltd., New Delhi.

6. Sinha, S.K. (1986). Reliability and Life Testing, Wiley Eastern Pvt. Ltd., New Delhi.

7. Yadavalli, V.S.S., Adendorff, K., Erasmus, G., Chandrasekhar, P., and Deepa, S.P. (2004). Confidence limits for expected waiting time of two queueing models, OriON (South Africa), 20, 1, 1-6.

8. Yadavalli, V.S.S., Natarajan, R., and Chandrasekhar, P. (2006). Confidence limits for the expected waiting time of $M / M / c / \infty$ and $M / M / c / N$ queueing models, Pak.J.Statist, 22, 2, 171-178. 